\title{
A novel clinical approach to evaluating changes in fat oxidation in healthy, overnight-fasted subjects
}

\author{
Stephanie A. Parsons ${ }^{1 *}$, Karen P. Jones ${ }^{1}$, Fanchao Yi ${ }^{1}$, Christopher P. Bock' ${ }^{1}$ Christopher J. Petucci ${ }^{2}$,
} Stella K. Betancourt ${ }^{2}$, Stephen J. Gardell ${ }^{2}$ and Steven R. Smith ${ }^{1,2}$

\begin{abstract}
Background: Obesity is characterized by impaired fat oxidation, and, therefore, the development of therapeutics designed to enhance fat oxidation for treating obesity is a growing focus in drug discovery. Evaluating such agents, however, is challenging in a clinical setting; requisite overnight fasting prior to a study visit increases whole body fat oxidation in lean, healthy subjects, making incremental increases potentially difficult to detect.

Methods: We recruited 16 lean/overweight participants and eight obese participants to evaluate novel methodology designed to optimally detect increases in fat oxidation. Carbohydrate intake was increased for three days leading up to the overnight test visit and evaluated as either a standalone strategy to suppress overnight fasting-related increases in fat oxidation or was combined with overnight administration of IV glucose in a subset of lean/overweight subjects. Indirect calorimetry was performed the following morning to examine the degree to which these interventions were able to diminish fasting related increases in fat oxidation. Fat oxidation was then subsequently stimulated by challenging patients with a lipid infusion to determine the importance of the preceding interventions on the ability to detect statistically significant increases in fat oxidation.

Discussion: In lean/overweight subjects, suppression of fasting-related increases in fat oxidation via increased dietary carbohydrate intake was not necessary to detect subsequent meaningful increases in fat oxidation rates following Intralipid infusion; however, a statistically significant decrease in respiratory quotient following Intralipid infusion could only be observed when the infusion was preceded with high carbohydrate dietary intake. Glucose infusion, which further suppressed fasting-related fat oxidation, in turn led to more dramatic increases in Intralipid-driven fat oxidation. Both lean/overweight and obese subjects could demonstrate statistically significant increases in fat oxidation following Intralipid infusion, a finding which supports the inclusion of obese subjects in similar future clinical studies. Lastly, serum levels of $\beta$-hydroxybutyrate correlated strongly with fat oxidation rates, as well as respiratory quotient, suggesting potential as a surrogate biomarker of fax oxidation. Taken together, we demonstrate the utility of novel methodology to facilitate measuring changes in fat oxidation in lean/ overweight and obese human volunteers in a clinical setting similar to that needed to test drugs designed to increase fat oxidation.
\end{abstract}

Keywords: Fat oxidation, Intralipid, Respiratory quotient, Hood calorimetry, Obese, Metabolic flexibility

\footnotetext{
* Correspondence: Stephanie.Parsons@flhosp.org

${ }^{1}$ Translational Research Institute for Metabolism and Diabetes, Florida

Hospital, 301 E. Princeton Street, Orlando, FL 32804, USA

Full list of author information is available at the end of the article
} 


\section{Background}

There is an ever growing need for new and innovative approaches for the treatment of obesity, and obesityrelated diseases (such as type 2 diabetes). Recent evidence suggests that impaired fat oxidation may be both a risk factor for the development of obesity as well as a barrier to effective weight loss [1-7]. Biological pathways that act to positively regulate fat oxidation and energy expenditure are, therefore, desirable targets for the design of therapeutic agents to combat obesity. Vital to the development of such compounds or biological agents, is the ability to test the efficacy of such agents in a clinical setting.

Increasing the flux of lipid into oxidative pathways is an attractive means to reverse metabolic diseases. First, reduced metabolic flexibility, a common hallmark of obesity, involves both impaired post-absorptive fat oxidation and decreased insulin stimulated glucose oxidation [8]. Toxic lipid intermediates are thought to produce insulin resistance at the cellular level [9-11]. Increasing fatty acid oxidation (FAOx) would presumably lead to a reduction of these intermediates and promote insulin action. Second, increased FAOx might create a negative energy balance. States of increased lipid supply and oxidation are associated with increased energy expenditure (EE) [12]. Increases in EE might offset the metabolic adaptation that occurs with weight loss $[13,14]$. Lastly, small changes in energy metabolism, over a long time frame and without compensatory changes in energy intake, can lead to an overall decrease in fat mass. This is thought to explain how increased habitual physical activity leads to better weight maintenance [15].

Early phase metabolic clinical studies typically center on safety and proof of concept, and often enlist lean healthy volunteers. The overnight fast that routinely precedes each study visit can make it challenging to detect changes in fat oxidation in this population. Healthy volunteers are generally highly metabolically flexible and will readily switch from carbohydrate to fat oxidation during an overnight fast $[7,8]$. Hence, under fasted conditions, these subjects already demonstrate higher than normal rates of fat oxidation, and any further stimulation of fat oxidation by a pharmacologic agent may be difficult to measure.

The purpose of this study was to evaluate novel approaches to mitigate fasting-related increases in fat oxidation in order to augment subsequent Intralipid-mediated increases in fat oxidation. These approaches were evaluated in both healthy lean or overweight and obese subjects in a setting typical of early phase clinical trials. In lean/ overweight individuals, we evaluated two approaches, a high carbohydrate diet with or without IV glucose, in order to determine if a nutrition-based strategy would be sufficient to adequately suppress fasting induced increases in fat oxidation, or if IV glucose throughout the overnight hours would be necessary to achieve this goal. We also examined the utility of this strategy in healthy obese individuals, the target population for fat oxidation-enhancing therapeutics. Additionally, we examined the potential utility of serum $\beta \mathrm{OHB}$ as a sensitive biomarker for evaluating changes in whole body fat oxidation. $\beta$-hydroxybutyrate $(\beta \mathrm{OHB})$, a metabolite produced by liver mitochondria during the oxidation of fatty acids, is upregulated during fasting or hypoglycemic conditions, as well as during infusion of lipid emulsions, such as Intralipid [16, 17]. Showing this metabolite to be a sensitive and robust biomarker of changes in fat oxidation could have implications for its inclusion in future clinical studies.

\section{Methods}

\section{Ethical approval and screening}

The study protocol was approved by the Florida Hospital Institutional Review Board and was carried out in accordance with the Declaration of Helsinki. Before taking part in the study, all subjects were evaluated for eligibility. All participants provided their written consent to take part in the study and were aware that they were free to withdraw from the experiments at any point. Procedures during the screening visit included the informed consent process, measurement of height and weight, Body Mass Index (BMI) calculation, waist measurement, vital signs (heart rate, blood pressure), electrocardiogram (ECG), medical history and physical, report of concomitant medications, lab work (complete blood count, comprehensive metabolic profile [consisting of tests for albumin, alkaline phosphatase, alanine aminotransferase, aspartate aminotransferase, blood urea nitrogen, calcium, chloride, carbon dioxide, creatinine, glucose, potassium, sodium, total bilirubin, total protein], CK, TSH, CRP, urinalysis, lipid profile [LDL, HDL, triglycerides], and serum beta hCG [for women of childbearing potential]).

\section{Subjects}

Twenty-four healthy men and women between the ages of 19 and 54 years of age took part in this study. Participants included 16 lean or overweight subjects (BMI $\geq$ $18-<30$ ) and eight obese subjects (BMI 30-35, inclusive). Female subjects were of non-childbearing potential. All were in good health and without evidence or history of clinically significant hematological, renal, endocrine, pulmonary, gastrointestinal, cardiovascular, hepatic, psychiatric, neurologic, allergic (including drug allergies, but excluding untreated, asymptomatic, seasonal allergies at time of dosing), muscle disease, diabetes, or severe uncontrolled hypertension. 


\section{Experimental protocol}

During a baseline visit occurring within two weeks prior to the overnight test visit, a frequently-sampled intravenous glucose tolerance test (FSIGTT) was performed following an overnight fast. For three days prior to the overnight test visit, all subjects were instructed to consume a diet high in carbohydrates. Ahead of this, subjects received nutritional counsel from nursing staff and were given examples of high carbohydrate foods (e.g., pasta, rice, potatoes) and were shown how to determine carbohydrate content from nutrition labels. Participants were instructed simply to consume greater than $150 \mathrm{~g}$ carbohydrates per day. No other standardization of dietary intake was imposed. For the overnight test visit, subjects were admitted to the research unit in the late afternoon. At approximately 18:00 subjects consumed a final high carbohydrate evening meal (either chicken parmesan with pasta and a side salad, or spaghetti with meatballs and side salad, and a side of bread), followed by a snack (package of graham crackers and cup of $2 \%$ milk) at 22:00.

Lean/overweight healthy subjects were randomized into Group 1 or Group 2.

\section{Group 1: Lean/overweight subjects receiving overnight saline infusion (designated LS) \\ Group 2: Lean/overweight subjects receiving overnight glucose infusion (designated LG) \\ Group 3: Consisted of obese subjects receiving overnight saline (designated OS)}

Intravenous glucose (10 \% Dextrose in Water, or D10W) or normal saline infused was infused at a rate of $125 \mathrm{ml} / \mathrm{h}$ from 22:00 until approximately 11:45 the following morning (see Fig. 1). This rate was determined as follows: The energy requirements $(\mathrm{kcal})$ were determined based on the needs of a $70 \mathrm{~kg}$ male consuming a standard American diet consisting of $35 \%$ fat, $15 \%$ protein, $50 \%$ carbohydrates. We then determined the amount of carbohydrate needed to completely replace carbohydrate oxidation of this prototypical subject at rest. Those requirements informed the glucose infusion rate $(4 \mathrm{kcal} / \mathrm{g}$ glucose). This rate was intended to provide sufficient calories to support REE (less the obligatory protein oxidation rate), increase insulin (and therefore suppress lipolysis), facilitate lipid storage through the increase in circulating insulin, and decrease lipid supply and oxidation. Additionally, we anticipated a reduction in FFA levels during the glucose infusion.

Immediately after stopping the glucose or saline infusion, Intralipid $20 \%$ infusion (Manufactured by Fresenius Kabi, Uppsala, Sweden, for Baxter Healthcare Corporation, Deerfield, IL http://www.accessdata.fda.gov/drugsatfda_docs/ label/2007/017643s072,018449s039lbl.pdf) was started at a rate of $1.5 \mathrm{ml} / \mathrm{min}$. The duration of Intralipid infusion was $3 \mathrm{~h}$.

Selected unblinded study staff verified, via two-nurse validation, that the test visit IV infusion (saline vs glucose) was appropriately indicated and administered according to the assigned randomization treatment. Any unblinded source documentation was secured in manila envelopes, which were then, distributed and managed only by unblinded study staff. Study blind was also maintained to the participant and all other study staff by obscuring the infusion bag and the content labeling.

\section{Frequently sampled intravenous glucose tolerance test} Bilateral antecubital IV lines were inserted, and $300 \mathrm{mg} / \mathrm{kg}$ glucose was injected at time $=0 \mathrm{~min}$, followed by collection of blood samples $(4 \mathrm{cc})$ at $1-2 \mathrm{~min}$ intervals until time $=$ $20 \mathrm{~min}$. At time $=20 \mathrm{~min}$, a bolus of insulin $(0.03 \mathrm{U} / \mathrm{kg}$ or $0.015 \mathrm{U} / \mathrm{kg}$ if the subject was considered lean and likely to be very insulin sensitive) was given via the IV line, and frequent sampling resumed for another $10 \mathrm{~min}$. For the remainder of the test (180 min), blood samples were collected every 10-20 min. Each blood sample was analyzed for glucose, insulin, and free fatty acid (FFA). These data were then submitted for calculation of the insulin sensitivity index $\left(\mathrm{S}_{\mathrm{I}}\right)$ and glucose effectiveness $\left(\mathrm{S}_{\mathrm{G}}\right)$ using the Minimal Model method of Bergman [18]. The minimal model analysis was accomplished using a program developed for the personal computer (MINMOD-Millennium, copyright R. Bergman), currently run in the PI's laboratory. The FSIGTT was then performed with the addition of exogenous insulin injection.

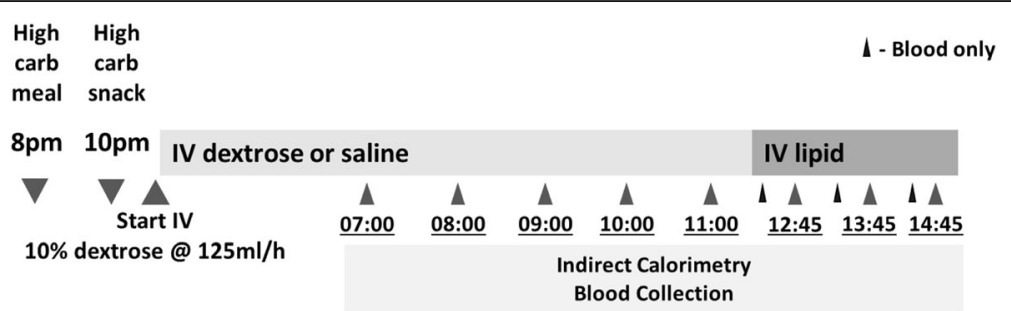

Fig. 1 Overview of Study Activities. Overview of timing of measurements and sample collection that occurred within this study 


\section{Test visit blood collections}

Blood samples were collected as depicted in Fig. 1. During the overnight test visit, blood samples were collected hourly (starting at 07:00) for measurement of glucose (YSI 2300 Stat Plus, Yellow Springs, OH), insulin (Meso Scale Discovery, Human Insulin, Rockville, MD), free fatty acids (WAKO NEFA - HR(2), Wako Life Sciences, Inc., Mountain View, CA), and triglycerides (Wako LType Triglyceride M, Wako Diagnostics, Richmond, VA) - all measured at The Translational Research Institute for Metabolism and Diabetes (TRI-MD).

Samples were also collected for measurement of serum $\beta$-hydroxybutyrate $(\beta \mathrm{OHB})$. $\beta \mathrm{OHB}$ was quantitated by GC/MS (ThermoFisher Trace GC Ultra/DSQ II single quadrupole mass spectrometer) in the Metabolomics Core at the Sanford Burnham Prebys Medical Discovery Institute. Calibration standards $(1-1000 \mu \mathrm{M})$ were prepared in water and spiked with ${ }^{13} \mathrm{C} 3-\beta \mathrm{OHB}(500 \mu \mathrm{M})$ as an internal standard. $\beta \mathrm{OHB}$ was extracted from plasma using a series of extraction and derivatization steps to form the corresponding trimethylsilyl derivative. Detection of the derivatized $\beta-\mathrm{OHB}$ was achieved by single ion monitoring after GC separation [19]. $\beta O H B$ levels were measured at two timepoints during the glucose or saline infusion period (09:30 and 11:30), as well as at the end of the Intralipid infusion period (15:00).

\section{DEXA}

Body composition was measured using a GE Lunar iDXA, running enCORE software version 13.3 (GE Medical Systems, Bucks, UK). All DEXA scans were analyzed in the Imaging Core using enCORE software to determine lean soft tissue mass (g), fat mass, including visceral adipose tissue, (g), and bone mass ( $\mathrm{g}$ ) of standard body regions, including leg, arm and trunk compositions. Test-retest CVs average $0.7 \%$ across a range of body composition.

\section{Indirect calorimetry \\ Baseline visit (During FSIGTT)}

Following IV line insertions and a 45 min rest period, indirect calorimetry was performed for 20 min each (once fasting, and once post glucose and insulin administration starting at $40 \mathrm{~min}$ post glucose injection) during the Baseline 1 FSIGTT. Respiratory quotient was measured using a MAX II Metabolic cart (AEI Technologies, Naperville, IL). The instrument was calibrated before each subject with standardized gases containing $5 \%$ $\mathrm{CO}_{2}$ and $95 \% \mathrm{O} 2$. Subjects were instructed to lie in a Semi-Fowler's position (supine position with the head of the bed positioned at approximately $30-45^{\circ}$ ) and remain motionless and awake during these periods. A transparent plastic hood connected to the device was placed over the head of the subject. Calculations of $\mathrm{O}_{2}$ consumption $\left(\mathrm{VO}_{2}\right)$ and $\mathrm{CO}_{2}$ production $\left(\mathrm{VCO}_{2}\right)$ were made from continuous measurements of $\mathrm{CO}_{2}$ and $\mathrm{O}_{2}$ concentrations in inspired and expired air diluted in a constant air flow $(\sim 40 \mathrm{~L} / \mathrm{min})$. The respiratory quotient (RQ) is defined by the ratio $\mathrm{VCO}_{2} / \mathrm{VO}_{2}$ and is an index of substrate use by the body; the relative volumes depend on the fuel source being metabolized. Respiratory coefficients range from about 1.0 (pure carbohydrate oxidation) to about 0.7 (pure fat oxidation). RQ was determined during an initial study baseline visit (within a 2 week window of the subsequent overnight test visit, no restrictions on macronutrient composition of the free-living diet). Prior to admission, dietary carbohydrate was increased as described in the Experimental Protocol. RQ was again measured during the test visit, following overnight infusion of either saline (LS, OS) or glucose (LG). Upon waking, the infusion continued, and RQ values were measured in hourly intervals.

\section{Test visit}

Following awakening and measurement of supine vitals, indirect calorimetry was performed for $30 \mathrm{~min}$ following a $30 \mathrm{~min}$ rest period, with the first $10 \mathrm{~min}$ of data discarded to ensure accuracy of the resting measurement. Indirect calorimetry was performed every hour thereafter. The rates of lipid oxidation were calculated as described by Elia and Livesey [20].

\section{Urine}

An overnight urine specimen was collected from bedtime to 06:30 for the measurement of urinary nitrogen and creatinine to calculate protein oxidation.

Fat oxidation rate was calculated based on the equations derived by Jequier et al. [21]: $1.689 \times \mathrm{VO} 2-1.689 \times$ VCO2 $-0.324 \times$ Protein Oxidation, where protein oxidation is calculated from Urinary Nitrogen (UN). Urine collection was not performed during the baseline visit and therefore the UN values from the following visit were used as an estimate of protein oxidation.

\section{Statistical analyses}

All data was analyzed either with Statistical Analysis System (SAS) or GraphPad Prism 6. Prior to performing data analysis, standard data screenings and audits were applied. These included screening demographic data for data entry errors, assessing the pattern of missing data, checking the distribution of each endpoints, and checking for outliers. For the description of baseline characteristics, continuous data were presented as the mean $+/$ - standard error and categorical data were summarized as frequencies and percentage by treatment groups. A two sample t-test was applied to check the difference of each characteristic between lean and obese subjects. The only exception was that the non-parametric Chi-square test 
was applied to test the sex proportional difference of two groups.

The potential effects of treatment, time (pre-/postIntralipid), and interaction were investigated using repeated measures ANOVA using SAS mixed procedure followed by post-hoc pairwise comparison (with stepdown Bonferroni or Tukey-Kramer correction). Pre-Intralipid data points were considered as baseline for each subject. If there were no statistical difference between these baseline data points, the pre-Intralipid values were expressed as the average of these points. For serum $\beta O H B$ values, logarithmic transformation was performed as the data were skewed; subsequently, correlations were performed. The results with a $P$ value $\leq 0.05$ were considered statistically significant.

\section{Results}

The Lean, Saline (LS) study population was comprised of five lean or overweight males and three lean or overweight females, Lean, Glucose (LG) group of six lean or overweight males and two lean or overweight females, and the Obese, Saline (OS) group of four obese males and four obese females. As stated, the Lean groups (LS, LG) are comprised of both lean and overweight subjects but are denoted as "L" for simplicity. Table 1 depicts the anthropometric and baseline laboratory values of the study populations. Values of all lean/overweight participants $(n=16)$ are used for comparison with the Obese group. The average age of the OS group was 43.7 years, significantly older than the average age of the Lean population. In addition, weight, BMI, total body mass, fat mass, \% body mass, and glucose values were all significantly greater in the Obese group. There were no significant differences in any of the values in Table 1 between the LS and LG groups.

Insulin sensitivity and insulin secretion were determined during the first baseline visit using an FSIGTT. There were no significant differences between the Lean and Obese cohorts or between the LS and LG groups.

\section{Glucose infusion reduces overnight fasting induced increases in fat oxidation}

Lean/overweight participants consuming an ad libitum diet exhibited an overnight fasted RQ of $0.861 \pm 0.01$. In

Table 1 Anthropometric and baseline laboratory values of the study population at screening

\begin{tabular}{|c|c|c|c|c|}
\hline & \multicolumn{2}{|l|}{ Lean } & \multirow{2}{*}{$\begin{array}{l}\text { Obese } \\
\text { Saline }\end{array}$} & \multirow{2}{*}{$\begin{array}{l}p \text {-value } \\
\text { Lean vs. obese }\end{array}$} \\
\hline & Saline & Glucose & & \\
\hline Age $(y r)$ & $35.7 \pm 3.9$ & $32.0 \pm 2.2$ & $43.7 \pm 3.8$ & 0.0272 \\
\hline $\operatorname{Sex}(M / F)$ & $5 / 3$ & $6 / 2$ & $4 / 4$ & $0.2059^{a}$ \\
\hline Height (cm) & $177.1 \pm 5.7$ & $182.2 \pm 1.8$ & $170.8 \pm 4.5$ & 0.1064 \\
\hline Weight (kg) & $81.8 \pm 4.9$ & $75.9 \pm 3.4$ & $95.3 \pm 3.9$ & 0.0001 \\
\hline $\mathrm{BMI}\left(\mathrm{kg} / \mathrm{m}^{2}\right)$ & $25.7 \pm 1.0$ & $22.7 \pm 1.2$ & $32.3 \pm 0.5$ & $<.0001$ \\
\hline Waist circumference (cm) & $87.1 \pm 3.7$ & $83.3 \pm 4.5$ & $106.3 \pm 4.5$ & 0.0005 \\
\hline Glucose (mg/dL) & $85.3 \pm 1.9$ & $84.3 \pm 1.5$ & $99.1 \pm 2.4$ & $<.0001$ \\
\hline Insulin (ulU/mL) & $61.7 \pm 12.9$ & $57.4 \pm 7.9$ & $52.9 \pm 8.9$ & 0.5917 \\
\hline Free Fatty Acids (mmol/L) & $0.57 \pm 0.06$ & $0.26 \pm 0.03$ & $0.43 \pm 0.04$ & 0.7864 \\
\hline Triglycerides (mg/dL) & $90.0 \pm 10.6$ & $118.7 \pm 30.5$ & $128.0 \pm 15.6$ & 0.3619 \\
\hline Thyroid-Stimulating Hormone (mlu/L) & $2.04+0.38$ & $1.69 \pm 0.21$ & $1.79 \pm 0.26$ & 0.8308 \\
\hline Creatine Phosphokinase (lu/L) & $89.8 \pm 14.2$ & $102.6 \pm 19.2$ & $123.8 \pm 19.8$ & 0.2145 \\
\hline \multicolumn{5}{|l|}{ DEXA } \\
\hline Total body mass (kg) & $81.5 \pm 6.8$ & $76.5 \pm 4.7$ & $96.5 \pm 5.7$ & 0.0219 \\
\hline Total lean mass (kg) & $52.3 \pm 5.2$ & $52.8 \pm 3.0$ & $54.7 \pm 4.8$ & 0.6907 \\
\hline Total fat mass (kg) & $26.1 \pm 2.9$ & $20.7 \pm 2.8$ & $38.6 \pm 1.8$ & 0.0001 \\
\hline Visceral adipose tissue mass (g) & $742.50 \pm 173.87$ & $518.63 \pm 222.32$ & $1262.63 \pm 167.13$ & 0.012 \\
\hline$\%$ Body fat & $32.3 \pm 3.1$ & $26.7 \pm 2.5$ & $40.7 \pm 2.1$ & 0.003 \\
\hline \multicolumn{5}{|l|}{ FSIGTT } \\
\hline Glucose effectiveness $\left(\mathrm{S}_{\mathrm{G}}\right)$ & $0.0201 \pm 0.0031$ & $0.0205 \pm 0.0043$ & $0.0165 \pm 0.0021$ & 0.344 \\
\hline Insulin sensitivity $\left(\mathrm{S}_{\mathrm{I}}\right)$ & $4.9 \pm 0.9$ & $5.4 \pm 0.8$ & $3.8 \pm 1.1$ & 0.2569 \\
\hline Acute insulin response to glucose $\left(A I R_{g}\right)$ & $385.1 \pm 110.8$ & $279.0 \pm 32.6$ & $497.4 \pm 177.4$ & 0.3883 \\
\hline
\end{tabular}

Data is represented as Mean \pm SEM; Bold: $P$ (two-sample t-test) $<0.05$

${ }^{\mathrm{a}}$ Chi squared test 
comparison, obese participants seemed to have an overall higher average fasting baseline RQ value $(0.895 \pm$ 0.026), but this difference was not found to be significant (Fig. 2a). All participants received a glucose challenge as part of a frequently sampled intravenous glucose tolerance test (FSIGTT). Results from this test determined that measures of glucose effectiveness, insulin sensitivity, and acute insulin response to glucose were similar between lean/overweight and obese participants (Table 1). Both lean/overweight and obese participants showed

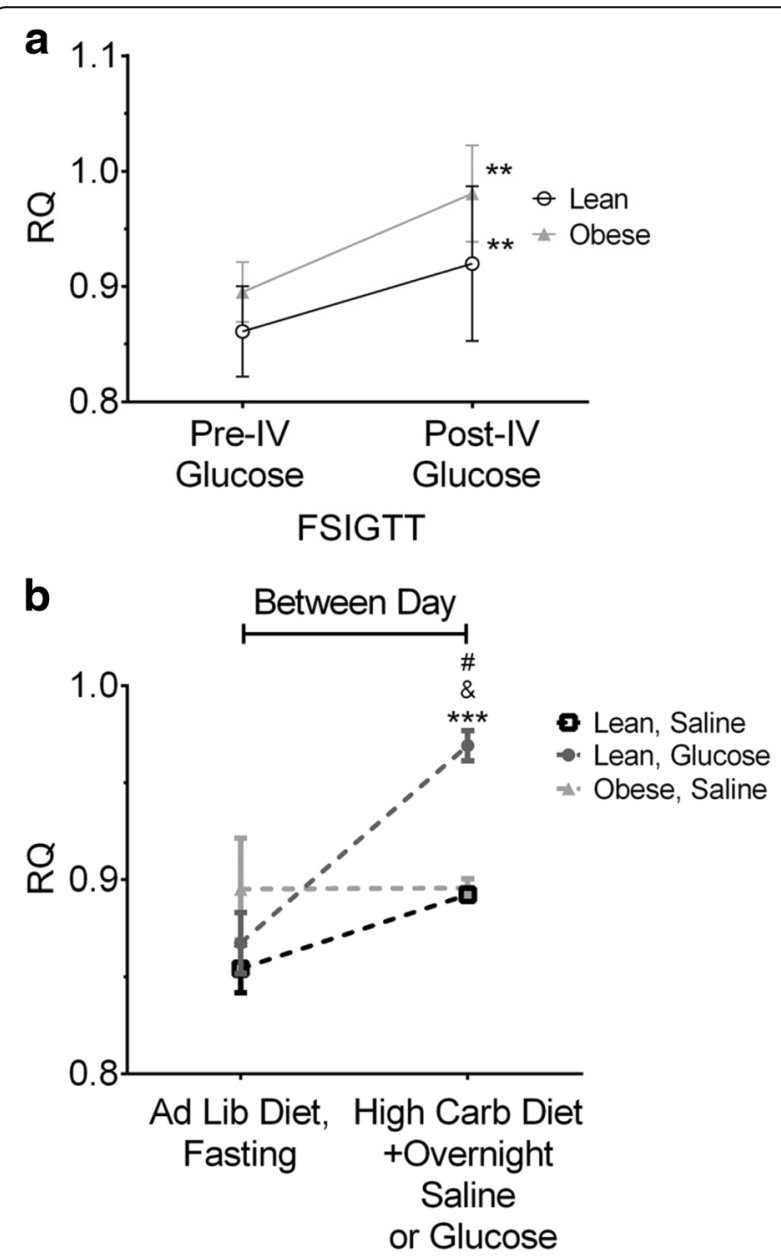

Fig. 2 Effect of Diet and Overnight Glucose Infusion on Fasting Respiratory Quotient Values. a RQ values obtained during the FSIGTT procedure, fasting versus post-glucose and insulin infusion. **Denotes significant difference within group between fasting RQ and $R Q$ measured post-glucose and insulin administration between the same group, ${ }^{* *} P=0.008$ obese and lean. $\mathbf{b}$ Comparison of fasting $\mathrm{RQ}$ obtained prior to the FSIGTT procedure (baseline study visit), to $\mathrm{RQ}$ measured during the Pre-Intralipid phase of the test visit. \#Denotes significant difference in LG group with respect to the LS group, $P=0.03$. \&Denotes significant difference in $L G$ group with respect to the OS group, $P=0.04$. ${ }^{* *}$ Denotes significant difference within LG group for RQ measured after high carbohydrate diet + overnight saline or glucose with respect to fasting RQ measured during ad libitum dietary intake, $P=0.0009$ similar upward shifts in their RQ values as measured between 40 and 60 min following glucose infusion, indicative of increased glucose oxidation in both groups. These increases were significant post- vs. pre-glucose challenge (Lean $0.920 \pm 0.017, P=0.008$; Obese $0.981 \pm$ $0.042, P=0.008$ ) (Fig. 2a).

As described in Methods, the test visit was preceded by three days of high carbohydrate dietary intake, culminating with a high carbohydrate meal and snack just prior to initiation of the overnight test visit infusion protocol (no further meals were consumed until the conclusion of the test visit the following day, $\sim 3$ p.m.). At approximately 23:00 on the night of the test visit, an antecubital IV was inserted, and IV glucose (LG) or normal saline (LS, OS) was infused overnight. Upon waking, the infusion continued, and RQ values were measured in hourly intervals. For the LS group, when comparing participants' average morning (08:00-11:00) RQ measurement to their fasted RQ measurement obtained immediately prior to the FSIGTT procedure, we observed that short term consumption of a high carbohydrate diet alone did not significantly increase RQ values, despite a slight observed increase $(0.859 \pm 0.013$ vs $0.892 \pm 0.002$; Fig. $2 b$ ). For the OS group, which demonstrated a higher baseline RQ under fasting conditions, no change in RQ could be observed with a high carbohydrate diet $(0.895 \pm 0.026$ vs $0.895 \pm 0.005)$. However, overnight infusion of glucose plus the high carbohydrate diet (LG) resulted in a significantly higher average RQ value as compared to fasting $R Q$ in the same group measured just prior to FSIGTT procedure (Ad Lib Diet, $0.868 \pm 0.016$ vs. $0.969 \pm 0.008 ; P=0.009$ ) (Fig. $2 b$ ). Average morning RQ values following overnight infusion for the LG group were also significantly higher than both the LS and OS groups ( $P=0.03$ vs. LS, $P=0.04$ vs. OS). These results indicate that in lean/overweight, healthy individuals, short term high carbohydrate dietary intake alone has no significant effect on RQ values following an overnight fast, but overnight glucose administration in combination with short term high carbohydrate results in significantly greater glucose oxidation as compared to overnight saline administration.

\section{Glucose and Intralipid infusion with or without dietary manipulation effects plasma metabolic parameters}

As with RQ, plasma levels of glucose, insulin, FFAs and triglycerides were measured at regular hourly intervals on the morning of the test visit, beginning at 08:00. It should be noted that there were no significant differences in any of these measurements at any of the timepoints analyzed. Therefore, for the purposes of comparison with postIntralipid levels, average values of these morning timepoints were used. Morning (also referred to as "pre-Intralipid") glucose levels were significantly elevated in participants in 
the OS group relative to those in the LS group $(P=0.006)$ (Fig. 3a). Participants in the LG group demonstrated the highest levels of blood glucose among all of the groups $(100.048 \pm 2.835 \mathrm{mg} / \mathrm{dL}$ vs. $83.956 \pm 2.204 \mathrm{mg} / \mathrm{dL}, \mathrm{LS}, P<$ 0.0001 and vs. $90.885 \pm 2.771 \mathrm{mg} / \mathrm{dL}, \mathrm{OS}, P<0.0001)$. Intralipid administration commenced at approximately 12:00. Following Intralipid administration, glucose levels in the LS group showed a slight downward trend toward the end of the Intralipid infusion period (Fig. 3a). In contrast, participants in the LG group, showed a dramatic decrease in blood glucose within the first $30 \mathrm{~min}$ of Intralipid infusion (from $100.048 \pm 2.835 \mathrm{mg} / \mathrm{dL}$ to $81.071 \pm$ $2.896 \mathrm{mg} / \mathrm{dL}, P<0.0001$, Fig. 3a). Changes in glucose levels in participants in the OS group were similar to those of the LS group (Fig. 3a).

Insulin levels showed similar patterns to glucose levels throughout the pre- and post-Intralipid infusion periods (Fig. 3b). Despite pre-Intralipid insulin levels appearing higher in participants in the OS group $(5.751 \pm 1.090$
$\mathrm{uUI} / \mathrm{mL})$ relative to those in the LS group $(3.127 \pm 0.236$ $\mathrm{uUI} / \mathrm{mL}$ ), however, this difference was not significant $(P=0.145)$. Participants in the LG group, though, did demonstrate significantly higher insulin levels relative to the other groups $(7.247 \pm 1.899 \mathrm{uUI} / \mathrm{mL}$ vs. LS, $P<$ 0.0001 and vs. OS, $P<0.0001$, Fig. $3 \mathrm{~b})$. Following Intralipid administration, insulin levels in participants in both the LS group and the OS group remained constant. Participants in the LG group, however, demonstrated dramatic significant decreases in insulin levels within the first $30 \mathrm{~min}$ following the switch from glucose to Intralipid infusion $(3.454 \pm 0.707 \mathrm{uUI} / \mathrm{mL}, \mathrm{P}=0.03)$, with levels remaining similar thereafter (Fig. 3b).

FFAs measured in the LG group were significantly lower relative to participants in the LS and OS groups $(0.056 \pm$ $0.005 \mathrm{mEq} / \mathrm{L}$ vs. $0.356 \pm 0.063 \mathrm{mEq} / \mathrm{L}, \mathrm{LS}, P<0.0001$, and vs. $0.269 \pm 0.033 \mathrm{mEq} / \mathrm{L}$, OS, $P=0.03$, Fig. $3 \mathrm{c}$ ). No significant difference in FFA levels were detected between LS and OS groups. During Intralipid administration, FFA



B
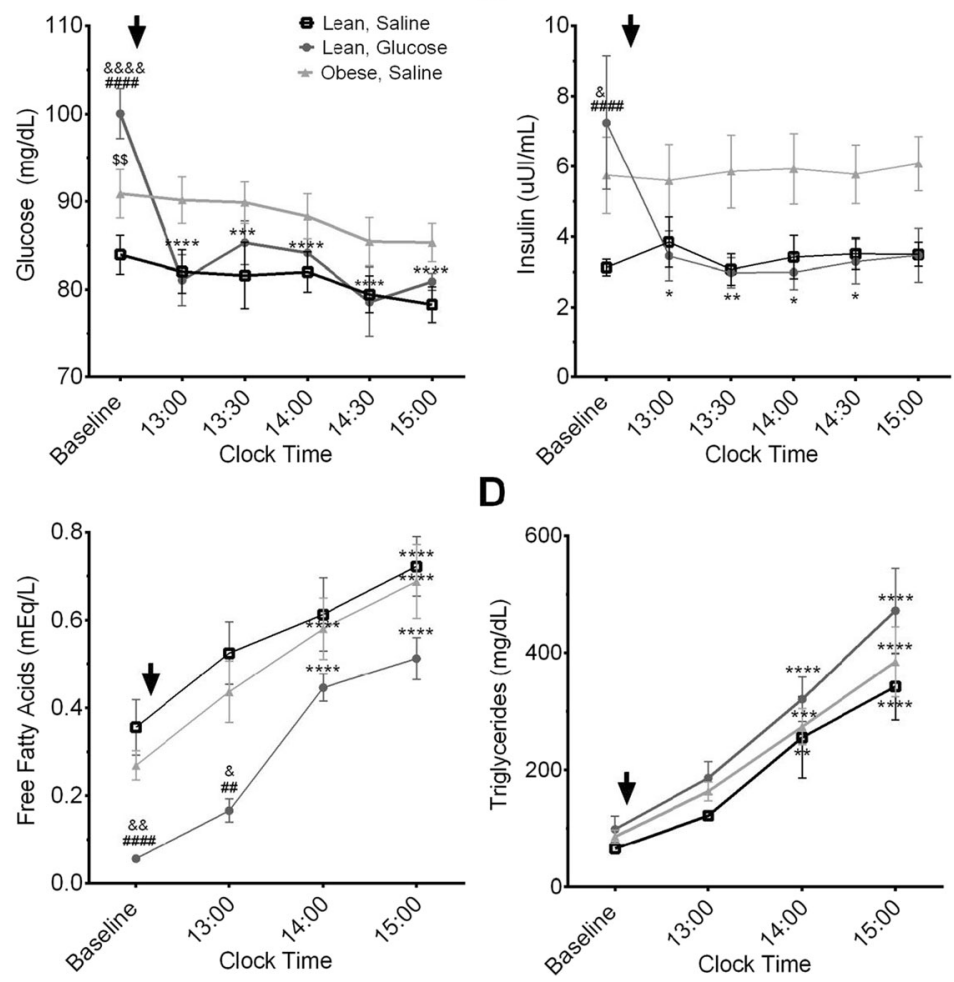

Fig. 3 Pre- and Post-Intralipid Glucose, Insulin, Triglycerides, and Free Fatty Acids Values. a Average baseline glucose levels measured during infusion of saline or glucose and glucose levels following Intralipid infusion. b Average baseline insulin levels measured during infusion of saline or glucose and insulin levels following Intralipid infusion. c Average baseline FFA levels measured during infusion of saline or glucose and FFA levels following Intralipid infusion. d Average baseline triglycerides levels measured during infusion of saline or glucose and triglycerides levels following Intralipid infusion. \$Denotes significant differences at the indicated timepoint in the LS group with respect to the OS group. \#Denotes significant differences at the indicated timepoint in the LG groups with respect to the LS group. \&Denotes significant differences at the indicated timepoint in the LG groups with respect to the OS group. *Denotes significant differences within group at the indicated Post-Intralipid timepoint with respect to the average Pre-Intralipid/Baseline measure of the same group. $\downarrow$ Arrow indicates that, for the LG subjects, the $10 \%$ IV glucose infusion was stopped at approximately 11:45. Note: For all symbols: ${ }^{*} P \leq 0.05,{ }^{*} P \leq 0.01,{ }^{* * *} P \leq 0.001,{ }^{* * *} P \leq 0.0001$ 
levels rose significantly in all groups, with the LG FFA levels ceasing to be significantly different from the LS and OS groups by $90 \mathrm{~min}$ post-Intralipid infusion (Fig. 3c).

No significant differences in triglyceride levels were observed prior to or following Intralipid infusion between any of the treatment groups (Fig. 3d). All groups demonstrated similar significant increases in triglyceride levels following infusion of Intralipid.

\section{Mitigation of fasting-related increases in fat oxidation enable better detection of changes in fat oxidation following intralipid infusion}

As mentioned, RQ was measured hourly throughout the morning and afternoon hours of the test visit. Within groups, RQ measurements were not significantly different throughout the morning hours (08:00-11:00) as saline or glucose infusion continued (Fig. 4a). It should be noted that in the OS group only, RQ measured at the

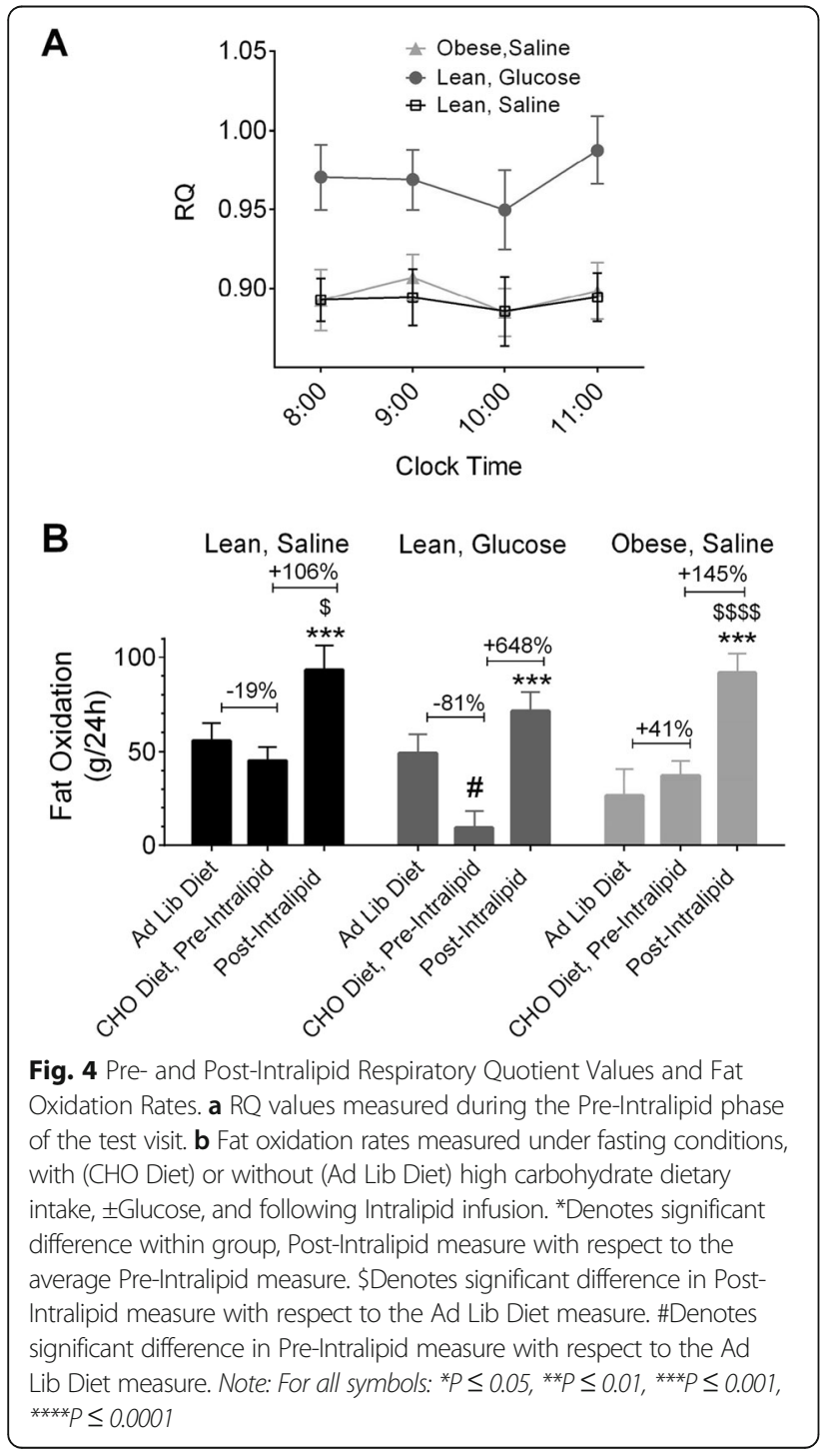

07:00 timepoint was significantly higher as compared to two of the other four a.m timepoints (data not shown). For this reason, average morning baseline values were determined for all groups using the 08:00-11:00 timepoints only. As described earlier, lean/overweight participants receiving IV glucose (LG) had significantly higher mean baseline RQ values than those receiving saline (Fig. 2b). Following the switch to Intralipid infusion, RQ values decreased sharply and significantly for all groups relative to Pre-Intralipid (Baseline) values (Additional file 1: Figure S1A). Notably, by 14:00, there ceased to be a difference between RQ values in the LG vs LS groups, but for the OS group this difference persisted at 15:00 (Additional file 1: Figure S1A).

In addition to respiratory quotient values, fat oxidation rates were calculated as described in Methods. Rates from participants during the pre-glucose infusion period that preceded the FSIGTT procedure (Ad Lib Diet) were compared to rates from those same participants obtained during the morning of the test visit, prior to the switch to Intralipid (CHO Diet, Pre-Intralipid). For the LS and OS groups, this comparison permits the examination of the high carbohydrate diet alone on lipid oxidation rates. We observed an average decrease in lipid oxidation rate in the LS group of $19 \%$ with high carbohydrate intake, which was not significant (Fig. 4b). Likewise, there was no effect of the high carbohydrate diet on fat oxidation rates in the OS group (Fig. 4b). The addition of the glucose infusion to the high carbohydrate intake in the LG group, however, effectively reduced lipid oxidation as evidenced by an average of $81 \%\left({ }^{\#} P=0.038\right)$. Following $2.5 \mathrm{~h}$ of Intralipid infusion, rates of fat oxidation increased significantly in all groups (Fig. 4b). Rates in the LS group increased by an average of $+106 \%$ and in the OS group by an average of $+145 \%$, relative to preIntralipid values, while the average change in the LG group was $+648 \%$ following the switch to Intralipid (Fig. 4b). Notably, the high carbohydrate diet was not necessary to detect subsequent significant increases in fat oxidation rates after Intralipid infusion in either the LS or OS groups (post-Intralipid vs ad lib diet, fasted, ${ }^{\$} P=0.034$, LS, and ${ }^{\$ \$ \$} P=0.0001$, OS, Fig. $\left.4 \mathrm{~b}\right)$.

When doing a similar comparison for RQ values measured prior to the baseline FSIGTT (Ad Lib Diet) to those measured following Intralipid infusion (Post-Intralipid), a $\triangle \mathrm{RQ}$ of $-3.78 \%$ and $-0.96 \%$ were observed for the lean/overweight groups (LS and LG, respectively, Table 2). For the OS group, which had a higher baseline (Ad Lib Diet) RQ than the lean/overweight groups, this decrease was significant. In contrast to what was observed for average fat oxidation rates in the LS group, however, the change in RQ between the ad lib diet and post-Intralipid timepoint was not significant. Furthermore, although the minor increase in RQ with consumption of a 
Table 2 Effect of nutrient intake and glucose/intralipid infusion on respiratory quotient

\begin{tabular}{lll}
\hline \multirow{2}{*}{ Group } & \multicolumn{2}{l}{ Mean percent change in RQ } \\
\cline { 2 - 3 } & $\begin{array}{ll}\text { Ad libitum, fasting } \\
\text { vs. post-intralipid }\end{array}$ & $\begin{array}{l}\text { High carb+/-glucose infusion } \\
\text { vs. post-intralipid }\end{array}$ \\
\hline Lean, Saline & $-3.8 \pm 2.0$ & $-\mathbf{7 . 9} \pm \mathbf{1 . 3}$ \\
Lean, Glucose & $-0.96 \pm 2.8$ & $-\mathbf{1 1 . 3} \pm \mathbf{2 . 0}$ \\
Obese, Saline & $\mathbf{- 1 0 . 4 3 \pm \mathbf { 3 . 3 }}$ & $\mathbf{- 1 0 . 4 9} \pm \mathbf{1 . 6}$ \\
\hline
\end{tabular}

$R Q$ respiratory quotient; Values are Mean \pm SEM, Bold denotes $P \leq 0.05$

high carbohydrate diet was itself also not significant (Additional file 1: Figure S1B), this increase did, however, facilitate a statistically significant $\Delta \mathrm{RQ}(-7.89 \%$, Table 2) between the CHO Diet and Post-Intralipid states in the LS group. Combining glucose infusion with the high carbohydrate diet resulted in an even greater $\triangle \mathrm{RQ}$ of $-11.44 \%$ in the LG group $(P<0.0001$; Table 2). In obese subjects, the post-Intralipid $\Delta R Q$ was significant with or without high carbohydrate dietary intake $(-10.43 \%$ compared to ad libitum diet fasting conditions and $-10.49 \%$ compared to fasting conditions post high carbohydrate consumption, Table 2). Graphs depicting the effect of each experimental condition on RQ in each group are presented in Additional file 1: Figure S1A, $\mathrm{S} 1 \mathrm{~B}$, and S1C.

\section{Serum levels of 3-hydroxybutyrate negatively correlate with RQ and positively correlate with fat oxidation}

Participants in the LS group demonstrated the highest PreIntralipid concentrations of $\beta \mathrm{OHB}(78.74 \pm 26.34 \mu \mathrm{M})$, while participants in the OS group exhibited intermediate levels $(23.70 \pm 5.97 \mu \mathrm{M})$, and participants in the LG group showed the lowest levels $(5.19 \pm 1.92 \mu \mathrm{M})$ (Fig. 5a). We investigated the potential significance of differences in preIntralipid $\beta \mathrm{OHB}$ levels among our groups by analyzing combined $\beta \mathrm{OHB}$ levels from both pre-Intralipid timepoints.


Fig. 5 Pre- and Post-Intralipid Levels of Serum $\beta$-Hydroxybutyrate. a Serum levels of $\beta O H B$ levels as measured during Pre-Intralipid phase of the test visit. b Correlation between average Pre-Intralipid serum $\beta O H B$ levels and average Pre-Intralipid RQ values. c Correlation between average Pre-Intralipid serum $\beta O H B$ levels and average Pre-Intralipid fat oxidation rates. $\mathbf{d}$ Average $\beta O H B$ serum levels measured during the Pre-Intralipid phase of the test visit and Post-Intralipid $\beta \mathrm{OHB}$ serum levels. ${ }^{*}$ Denotes significant difference within group at the Post-Intralipid timepoint with respect to the average Pre-Intralipid/Baseline measure for the same group $(P=0.005$, both). \#\#Denotes significance difference between $L S$ group and LG group with respect to the Post-Intralipid timepoint $(P=0.007)$ 
$\beta \mathrm{OHB}$ was not, however, found to be significantly different between any of the groups. However, analysis of average $\beta \mathrm{OHB}$ readings at the pre-Intralipid timepoints in all groups and their corresponding average RQ values or average fat oxidation rates revealed a significant negative correlation $(P=0.0075)$ between RQ and $\beta \mathrm{OHB}$ and a significant positive correlation $(R=0.0129)$ between fat oxidation rate and $\beta \mathrm{OHB}$ was found (Fig. $5 \mathrm{~b}$ and c, respectively). It is important to note that, for this analysis, log transformation of the $\beta \mathrm{OHB}$ data was performed in order to conform the skewness of the data. It should also be noted that correlation of pre- and postIntralipid $\mathrm{RQ}$ or fat oxidation rates and $\beta \mathrm{OHB}$ could not be determined due to skewing of the pre-Intralipid $\beta \mathrm{OHB}$ data.

Following Intralipid infusion, increases in $\beta \mathrm{OHB}$ were observed in all three groups relative to pre-Intralipid levels. These increases were significant for the LS $(P=$ $0.005)$ and $\mathrm{OS}(P=0.005)$ groups (Fig. $5 \mathrm{~d})$. Post-Intralipid levels of $\beta \mathrm{OHB}$ in the LS group remained significantly higher than LG group $\left({ }^{\# \#} P=0.007\right.$, Fig. $\left.5 \mathrm{~d}\right)$ and trended higher $(P=0.08)$ as compared to the OS group.

\section{Discussion}

Increased de novo lipogenesis and impaired fatty acid oxidation appear to be key in the development of obesity and insulin resistance. Therefore, enzymes in these pathways have generated keen interest as targets for therapeutic intervention. Such therapies, however, can encounter significant challenges when it comes to clinical testing. One of these challenges centers around the fact that fasting is a routine requirement of volunteers prior to a study visit. However, fasting alone is sufficient to increase fat oxidation in healthy subjects. This increase could very conceivably diminish the subsequent effects of the pharmacotherapy and make further increases related to the intervention less likely to be observed. Furthermore, detection of drugrelated increases in fat oxidation may require increased substrate availability. Our protocol takes into account these important considerations, and, as we have shown, demonstrates that, in a clinical setting under conditions typical of a phase 1 test visit, 1) changes in fat oxidation in human subjects can be measured in a sensitive and robust manner, and 2) statistically meaningful increases in fat oxidation can be observed.

Fundamental to our protocol design was the suppression of fasting-induced increased fat oxidation through a nutrition-based approach, either alone or in combination with intravenous infusion of glucose. We found that increasing the carbohydrate intake of both lean/overweight and obese participants through consumption of a high carbohydrate diet for a period of three days neither led to significantly higher fasting RQ values nor significantly decreased lipid oxidation, although minor shifts in the
LS group could be observed. However, infusion of glucose in combination with the high carbohydrate diet during the overnight hours of the test visit, did result in dramatic significant decreases in lipid oxidation and increased RQ.

Following overnight infusion of either saline or glucose, a switch to Intralipid infusion was initiated as a means to increase lipid availability and drive fat oxidation, and all groups demonstrated significant increases in fat oxidation in response to this lipid challenge. To better determine how essential the high carbohydrate diet, with or without glucose infusion, was to this observation, a comparison of participants' fasting RQ values and lipid oxidation rates (obtained during a baseline study visit, prior the FSIGTT procedure, ad libitum dietary intake), and these measures post-Intralipid infusion (test visit) were carried out. With regard to RQ, for lean/ overweight participants, the high carbohydrate diet alone proved necessary and sufficient. The modest increases in RQ owing to the carbohydrate intake were adequate to enable subsequent detection of statistically significant decreases in RQ in response to Intralipid infusion. However, when evaluating lipid oxidation rates, this was not found to be the case. In fact, significant increases in fat oxidation (LS group) with Intralipid could be detected without any dietary changes or IV glucose. However, the changes were less striking as compared to the observed with the glucose infusion. Taken together, these findings suggest that in future clinical studies, for lean or overweight subjects it may be prudent to implement measures to inhibit fasting-induced increases in fat oxidation subsequent to clinical testing of therapeutic agents to enhance fat oxidation. Furthermore, while not tested here, infusion of glucose without the accompanying high carbohydrate diet could be ideal approach to doing this when precise standardization of pre-testing nutrient intake proves impractical.

The high carbohydrate diet had no effect on lipid oxidation or RQ in obese subjects and was not necessary to observe statistically significant increases in fat oxidation, although the high carbohydrate diet did further increase the significance of $\triangle R Q$ pre- versus post-Intralipid. It has been reported previously that fasting RQ is elevated in at the whole body level in obese adolescents [22] and in subjects with a family history of type 2 diabetes [23]. While we consistently observed elevated fasting RQ/ lower rates of fat oxidation under ad libitum conditions in our obese participants relative to the lean/overweight participants, these differences did not prove to be significant. Perhaps the most noteworthy observation with this subject group, however, is their preservation of their metabolic flexibility to lipid and ability to adapt fat utilization to availability. This is important as these individuals represent the target population for pharmacotherapies designed 
to enhance fat oxidation. It, therefore, increases confidence that a pharmacodynamic response to therapeutics designed to enhance fat oxidation could be detected in the healthy obese population and greatly strengthens the rationale for their inclusion in early clinical studies.

Finally, we found that accompanying the decrease in RQ and increase in lipid oxidation with Intralipid infusion was corresponding increase in serum levels of $\beta O H B$. Increased circulating levels of ketones are a normal response to increased lipid flux to the liver from the adipose tissue; fatty acids are transformed in the mitochondria of liver and kidney cells to ketone units, including $\mathrm{BOHB}$, which then circulate back into the bloodstream. Significant increases in $\beta \mathrm{OHB}$ in response to Intralipid infusion in both the LS and OS groups were observed. Increases were also observed in the LG group, but the increase was not statistically significant, presumably due in part to a lag in $\beta \mathrm{OHB}$ production resultant from the prior glucose infusion. Furthermore, before infusion of Intralipid was even initiated, $\beta \mathrm{OHB}$ levels were clearly found to have a significant negative correlation with RQ and significant positive correlation with lipid oxidation. Serum $\beta \mathrm{OHB}$ may, therefore, have utility in the clinic as a secondary biomarker for measuring fat oxidation.

While the protocol presented here highlights several important considerations for the design of future clinical studies to measure changes in fat oxidation, our approach is not without certain limitations. For example, here we employ Intralipid infusion to drive increased lipid oxidation, perhaps making it most relevant with respect to testing of therapeutics that raise fatty acids to drive fat oxidation. To that point, however, the ability of indirect calorimetry to sensitively measure changes in relative macronutrient oxidation and energy expenditure is well-established, and our protocol serves simply to create an optimal experimental environment that potentiates the ability to reveal significant increases in fatty acid oxidation in those subjects that already have a high capacity for fatty acid oxidation. Unfortunately, we have neither a pharmacological agent available to serve as a positive control in our study, nor has a clinically meaningful change in fatty acid oxidation yet been defined, so it is not possible yet to put the changes in lipid oxidation that we see with our model into this context. "Real-world" testing of our model and assessment of the benefits of increased fatty acid oxidation will depend the development of these pharmacological agents and will permit us to fully test the metabolic flexibility hypothesis.

Another shortcoming of our study is limited data to support the observation that $\beta \mathrm{OHB}$ could be robust and sensitive biomarker of fat oxidation. While it is clear that dramatic increases in $\beta \mathrm{OHB}$ are associated with increases in fat oxidation (in general, a $10 \%$ reduction in $R Q$ is associated with about an $800 \%$ increase in $\beta \mathrm{OHB})$, statistical analysis of the pre- and post-Intralipid correlation of $\mathrm{RQ}$ and $\beta \mathrm{OHB}$ was not possible due to the skewness of the pre-Intralipid $\beta \mathrm{OHB}$ data; whether there is a significant correlation will, therefore, require further efforts to establish. Toward this end, the inclusion of graded Intralipid infusions may be helpful in better refining the relationships between lipid supply, oxidation, and biomarkers such as $\beta \mathrm{OHB}$ or acylcarnitines. It also merits consideration that biomarkers of increased oxidation driven by lipid excess may differ from biomarkers that relate to the activation of enzymes that consume fatty acids or from biomarkers that relate to increasing demand for substrate (such as uncoupling). This is an important potential distinction for future investigation.

In conclusion, while diet and behavior modifications will continue to be an important focus with regard to the treatment and management of obesity, the achievement of long-term, clinically relevant weight loss will likely be achieved only through the development of novel and innovative therapies, such as those that promote increased fat oxidation. The critical evaluation of such therapies will require equally innovative approaches in the clinic. Here we have demonstrated the utility of a novel approach designed to enable statistically significant changes in fatty acid oxidation to be measured in a clinical setting.

\section{Conclusion}

The pursuit of new anti-obesity pharmacotherapies is increasingly focused on strategies to target pathways important for the regulation of energy balance. Of particular interest is the modulation of enzymes that play a key role in mitochondrial $\beta$-oxidation. As these agents ascend into the clinic for evaluation, demonstration of their ability to promote fat oxidation will be crucial and likely require preemptive measures to diminish the effect of other factors, such as fasting or diet, that impact fat oxidation. The protocol described herein demonstrates the utility of these measures and may serve as a paradigm for such studies moving forward.

\section{Additional file}

\footnotetext{
Additional file 1: Figure S1. Pre- and Post-Intralipid Respiratory Quotient Values. A. Average RQ values measured during the Pre-Intralipid/Baseline phase of the test visit and post-Intralipid RQ values. \#Denotes significant difference in LG group Pre-Intralipid/Baseline measure with respect to the LS group. \&Denotes significant difference in LG group Pre-Intralipid/Baseline measure with respect to the OS group. ${ }^{*}$ Denotes significant difference within group, Post-Intralipid measure with respect to the average PreIntralipid/Baseline measure. B. RQ measured under fasting conditions, with (CHO Diet) or without (Ad Lib Diet) high carbohydrate dietary intake, \pm Glucose, and following Intralipid infusion. *Denotes significant difference within group, Post-Intralipid measure with respect to the average Pre-Intralipid measure. \$Denotes significant difference in Post Intralipid measure with respect to the Ad Lib Diet measure. \#Denotes
} 
significant difference in Pre-Intralipid measure with respect to the Ad Lib Diet measure. C. Comparison of fasting RQ values ("Between Day") and $R Q$ values obtained during the saline or glucose infusion phase of the test visit ("Within Day") to post-Intralipid RQ values. *Denotes significant difference within group, Post-Intralipid measure with respect to the Pre-Intralipid/Baseline measure. Note: For all symbols: ${ }^{*} P \leq 0.05,{ }^{* * P} \leq 0.01,{ }^{* * *} P \leq 0.001,{ }^{* * * *} P \leq 0.0001$ (DOCX $667 \mathrm{~kb})$

\section{Abbreviations}

Beta hCG: Beta human chorionic gonadotropin; BMl: Body mass index; CBC: Complete blood count; CHO: Carbohydrate; CK: Creatinine kinase; CMP: Comprehensive metabolic profile; CRP: C reactive protein; DBP: Diastolic blood pressure; ECG: Electrocardiogram; EE: Energy expenditure; FAOx: Fatty acid oxidation; FSIGTT: Frequently-sampled intravenous glucose tolerance test; HDL: High density lipoprotein; HR: Heart rate; IV: Intravenous; LDL: Low density lipoprotein; LG: Lean, glucose; LS: Lean, saline; OS: Obese, saline; RQ: Respiratory quotient; SBP: Systolic blood pressure; TSH: Thyroid-stimulating hormone; $\mathrm{VCO}_{2}: \mathrm{CO}_{2}$ production; $\mathrm{VO}_{2}: \mathrm{O}_{2}$ consumption; $\beta \mathrm{OHB}$ : $\beta$-hydroxybutyrate

\section{Acknowledgments}

We wish to thank Takeda Pharmaceuticals for their financial support and intellectual input in study design. We would also like to thank Hui Xie for his assistance with the statistical analysis of data presented in this study. This study was made possible through the efforts and dedication of TRI-MD staff and the study volunteers. We wish to extend our gratitude all that participated in this study.

\section{Funding}

This research was funded through a partnership with Takeda Pharmaceuticals. The Southeast Center for Integrated Metabolomics (NIH Grant U24 DK097209-01A1) provided support regarding generation of $\beta O H B$ data included in this study.

\section{Authors' contributions}

SS conceived the study concept and design, obtained funding, and reviewed/edited the manuscript. SP and SS are the guarantors of this work and, such, had full access to all the data in the study and takes responsibility for the integrity of the data and the accuracy of the data analysis. SP wrote the manuscript, elaborated tables and figures, participated in the analysis and interpretation of data. FY participated in data analysis. CP, SG, and SKB acquired data and reviewed/edited the manuscript. All authors read and approved the final manuscript.

\section{Competing interests}

SRS and SP received research support from Takeda Pharmaceuticals, Inc. The other authors have no conflicts of interest. None of the funding sources played a role in the collection, analysis, or interpretation of the data or in the decision to submit the manuscript for publication.

\section{Consent for publication}

Not Applicable.

\section{Ethics approval and consent to participate}

The Institutional Review Board at Florida Hospital approved this research for ethical standards, scientific merit, and regulatory compliance (IRBNet\# 241688-23). The Office of Research Administration (ORA) provided support and oversight to ensure integrity of this research at Florida Hospital.

\section{Author details}

${ }^{1}$ Translational Research Institute for Metabolism and Diabetes, Florida Hospital, 301 E. Princeton Street, Orlando, FL 32804, USA. ${ }^{2}$ Metabolomics Core, Sanford Burnham Prebys Medical Discovery Institute, Orlando, FL 32827, USA.

Received: 8 June 2016 Accepted: 15 September 2016 Published online: 06 October 2016

\section{References}

1. Chen MB, McAinch AJ, Macaulay SL, Castelli LA, O'Brien PE, Dixon JB, CameronSmith D, Kemp BE, Steinberg GR. Impaired activation of AMP-kinase and fatty acid oxidation by globular adiponectin in cultured human skeletal muscle of obese type 2 diabetics. J Clin Endocrinol Metab. 2005;90:3665-72.

2. Kelley DE, Goodpaster B, Wing RR, Simoneau JA. Skeletal muscle fatty acid metabolism in association with insulin resistance, obesity, and weight loss. Am J Physiol. 1999;277:E1130-41.

3. Bandyopadhyay GK, Yu JG, Ofrecio J, Olefsky JM. Increased malonyl-CoA levels in muscle from obese and type 2 diabetic subjects lead to decreased fatty acid oxidation and increased lipogenesis; thiazolidinedione treatment reverses these defects. Diabetes. 2006;55:2277-85.

4. Simoneau JA, Veerkamp JH, Turcotte LP, Kelley DE. Markers of capacity to utilize fatty acids in human skeletal muscle: relation to insulin resistance and obesity and effects of weight loss. FASEB J. 1999:13:2051-60.

5. Heilbronn L, Smith SR, Ravussin E. Failure of fat cell proliferation, mitochondrial function and fat oxidation results in ectopic fat storage, insulin resistance and type II diabetes mellitus. Int J Obes Relat Metab Disord. 2004;28 Suppl 4:S12-21.

6. Ukropcova B, McNeil M, Sereda O, de Jonge L, Xie H, Bray GA, Smith SR. Dynamic changes in fat oxidation in human primary myocytes mirror metabolic characteristics of the donor. J Clin Invest. 2005;115:1934-41.

7. Ukropcova B, Sereda O, de Jonge L, Bogacka I, Nguyen T, Xie H, Bray GA, Smith SR. Family history of diabetes links impaired substrate switching and reduced mitochondrial content in skeletal muscle. Diabetes. 2007;56:720-7.

8. Kelley DE, Mandarino L. Fuel selection in human skeletal muscle in insulin resistance: a reexamination. Diabetes. 2000:49:677-83.

9. Amati F. Revisiting the diacylglycerol-induced insulin resistance hypothesis. Obes Rev. 2012:13 Suppl 2:40-50.

10. Samad F, Badeanlou L, Shah C, Yang G. Adipose tissue and ceramide biosynthesis in the pathogenesis of obesity. Adv Exp Med Biol. 2011;721:67-86

11. Schooneman MG, Vaz FM, Houten SM, Soeters MR. Acylcarnitines: reflecting or inflicting insulin resistance? Diabetes. 2013;62:1-8.

12. Schiffelers $S L$, Saris $W H$, van Baak MA. The effect of an increased free fatty acid concentration on thermogenesis and substrate oxidation in obese and lean men. Int J Obes Relat Metab Disord. 2001;25:33-8.

13. Heilbronn LK, de Jonge L, Frisard MI, DeLany JP, Larson-Meyer DE, Rood J, Nguyen T, Martin CK, Volaufova J, Most MM, et al. Effect of 6-month calorie restriction on biomarkers of longevity, metabolic adaptation, and oxidative stress in overweight individuals: a randomized controlled trial. JAMA. 2006:295:1539-48

14. Rosenbaum M, Hirsch J, Gallagher DA, Leibel RL. Long-term persistence of adaptive thermogenesis in subjects who have maintained a reduced body weight. Am J Clin Nutr. 2008;88:906-12.

15. Hall KD, Sacks G, Chandramohan D, Chow CC, Wang YC, Gortmaker SL, Swinburn BA. Quantification of the effect of energy imbalance on bodyweight. Lancet. 2011:378:826-37.

16. Amery CM, Round RA, Smith JM, Nattrass M. Elevation of plasma fatty acids by ten-hour intralipid infusion has no effect on basal or glucose-stimulated insulin secretion in normal man. Metabolism. 2000;49:450-4.

17. Jensen CB, Storgaard H, Holst JJ, Dela F, Madsbad S, Vaag AA. Insulin secretion and cellular glucose metabolism after prolonged low-grade intralipid infusion in young men. J Clin Endocrinol Metab. 2003:88:2775-83.

18. Boston RC, Stefanovski D, Moate PJ, Sumner AE, Watanabe RM, Bergman RN. MINMOD Millennium: a computer program to calculate glucose effectiveness and insulin sensitivity from the frequently sampled intravenous glucose tolerance test. Diabetes Technol Ther. 2003:5:1003-15.

19. Haqq AM, Lien LF, Boan J, Arlotto M, Slentz CA, Muehlbauer MJ, Rochon J, Gallup D, McMahon RL, Bain JR, et al. The Study of the Effects of Diet on Metabolism and Nutrition (STEDMAN) weight loss project: Rationale and design. Contemp Clin Trials. 2005;26:616-25

20. Elia M, Livesey $\mathrm{G}$. Energy expenditure and fuel selection in biological systems: the theory and practice of calculations based on indirect calorimetry and tracer methods. World Rev Nutr Diet. 1992;70:68-131.

21. Jequier $E$, Acheson $K$, Schutz $Y$. Assessment of energy expenditure and fuel utilization in man. Annu Rev Nutr. 1987;7:187-208.

22. Perseghin G, Bonfanti R, Magni S, Lattuada G, De Cobelli F, Canu T, Esposito A, Scifo P, Ntali G, Costantino F, et al. Insulin resistance and whole body energy 
homeostasis in obese adolescents with fatty liver disease. Am J Physiol Endocrinol Metab. 2006;291:E697-703.

23. Lattuada G, Costantino F, Caumo A, Scifo P, Ragogna F, De Cobelli F, Del Maschio A, Luzi L, Perseghin G. Reduced whole-body lipid oxidation is associated with insulin resistance, but not with intramyocellular lipid content in offspring of type 2 diabetic patients. Diabetologia. 2005;48:741-7.

Submit your next manuscript to BioMed Central and we will help you at every step:

- We accept pre-submission inquiries

- Our selector tool helps you to find the most relevant journal

- We provide round the clock customer support

- Convenient online submission

- Thorough peer review

- Inclusion in PubMed and all major indexing services

- Maximum visibility for your research

Submit your manuscript at www.biomedcentral.com/submit
Biomed Central 